\title{
The New Infinite Sequence Solutions of Multiple Sine-Gordon Equations
}

\author{
Yu Mei Bai1, Taogetusang1,2 \\ ${ }^{1}$ The College of Mathematical, Inner Mongolia University for Nationalities, Tongliao, China \\ ${ }^{2}$ College of Mathematical Science, Inner Mongolia Normal University, Huhhot, China \\ Email: baiym2008@sohu.com,tgts@imnu.edu.cn
}

Received 15 March 2016; accepted 25 April 2016; published 28 April 2016

Copyright (C) 2016 by authors and Scientific Research Publishing Inc.

This work is licensed under the Creative Commons Attribution International License (CC BY). http://creativecommons.org/licenses/by/4.0/

(c) () Open Access

\begin{abstract}
By the function transformation and the first integral of the ordinary differential equations, the problem of solving the solutions of the double sine-Gordon equation and the treble sine-Gordon equation is researched, and the new solutions are obtained. First, the problem of solving the solutions of the double sine-Gordon equation and the treble sine-Gordon equation is changed to the problem of solving the solutions of the nonlinear ordinary differential equation. Second, with the help of the Bäcklund transformation and the nonlinear superposition formula of solutions of the first kind of elliptic equation and the Riccati equation, the new infinite sequence soliton-like solutions of two kinds of sine-Gordon equations are constructed.
\end{abstract}

\section{Keywords}

First Integral, Multiple Sine-Gordon Equation, Bäcklund Transformation, New Infinite Sequence Soliton-Like Solutions

\section{Introduction}

Refs. [1]-[3] studied the problem of solving the solutions of the double sine-Gordon equation, and a finite number of new solutions consisting of Jacobi elliptic function, hyperbolic function and trigonometric function are obtained.

$$
u_{x t}=p[\sin (u)+2 \lambda \sin (2 u)]
$$

where $p$ and $\lambda$ are constants.

Refs. [4] [5] obtained the new solutions consisting of Jacobi elliptic function, hyperbolic function and trigonometric function of the treble sine-Gordon equation. 


$$
u_{x x}-u_{t t}=\alpha \sin (u)+\frac{1}{3} \beta \sin \left(\frac{1}{3} u\right)+\frac{2}{3} \gamma \sin \left(\frac{2}{3} u\right)
$$

where $\alpha, \beta$ and $\gamma$ are constants.

In this paper, by the function transformation and the first integral of the ordinary differential equations, the problem of solving the solutions of the double sine-Gordon equation and the treble sine-Gordon equation is changed to the problem of solving the solutions of the nonlinear ordinary differential equation. Based on these, with the help of the Bäcklund transformation and the nonlinear superposition formula of solutions of the first kind of elliptic equation and the Riccati equation, the new infinite sequence soliton-like solutions of two kinds of sine-Gordon equations are constructed, which are consisting of Riemann $\theta$ function, Jacobi elliptic function, hyperbolic function, trigonometric function and rational function.

\section{Some Kinds of New Conclusions of Nonlinear Ordinary Differential Equation}

The relative conclusions of the Bäcklund transformation of some kinds of ordinary differential equations introduced as follows are very important in constructing the new solutions of the two kinds of sine-Gordon equations.

$$
\left(\frac{\mathrm{d} z(\xi)}{\mathrm{d} \xi}\right)^{2}=\left(z^{\prime}(\xi)\right)^{2}=d+a z^{2}(\xi)+b z^{4}(\xi)+c z^{6}(\xi) .
$$

Theorem 2.1 When $d=0$, there is the fitting Bäcklund transformation (4) between the ordinary differential Equation (3) and the Riccati Equation (5).

$$
\begin{gathered}
z^{2}(\xi)=\frac{\Psi^{2}(\xi)-a}{b-2 \sqrt{c} \Psi(\xi)}, \\
\frac{\mathrm{d} \Psi(\xi)}{\mathrm{d} \xi}=\Psi^{\prime}(\xi)=\Psi^{2}(\xi)-a .
\end{gathered}
$$

According to the relative conclusions of the Riccati Equation [6] and the fitting Bäcklund transformation (4), the solutions of the ordinary differential Equation (3) $(d=0)$ are obtained.

Theorem 2.2 There is the following fitting Bäcklund transformation between the ordinary differential Equation (3) and the first kind of elliptic Equation (6).

Then we put forward the fitting Bäcklund transformation between the ordinary differential Equation (3) and the first kind of elliptic Equation (6) in some cases.

$$
\left(\frac{\mathrm{d} y(\xi)}{\mathrm{d} \xi}\right)^{2}=\left(y^{\prime}(\xi)\right)^{2}=A+B y^{2}(\xi)+C y^{4}(\xi) .
$$

Case 1. When $d=-\frac{1}{2 P}\left(B R-\frac{\sqrt{2 B C R^{3}}}{\sqrt{Q}}\right), a=\frac{1}{4 Q}(6 C R-6 \sqrt{2 B C R Q}+B Q)$, $b=\frac{\sqrt{C} P}{2 \sqrt{Q^{3}}}\left(\frac{2 C \sqrt{2 R}}{\sqrt{B}}-4 \sqrt{C Q}+\frac{\sqrt{2 B} Q}{\sqrt{R}}\right), \quad c=\frac{C P^{2}}{4 B R Q^{2}}(2 C R-2 \sqrt{2 B C Q R}+B Q), \quad A=-\frac{R}{Q^{2}}(C R-B Q)$ there is the following Bäcklund transformation between the ordinary differential Equation (3) and the first kind of elliptic Equation (6).

$$
z^{2}(\xi)=\frac{B P R \sqrt{C}+\sqrt{B R}\left[\left(-C P^{2}(2 C R+2 \sqrt{2 B C R Q}+B Q)\right)^{\frac{1}{2}}-C P \sqrt{2 Q} y(\xi)\right] y(\xi)}{P^{2} \sqrt{C}\left[B+2 C y^{2}(\xi)\right]} .
$$

Case 2. When $d=-\frac{1}{2 P}\left(B R+\frac{\sqrt{2 B C R^{3}}}{\sqrt{Q}}\right), a=\frac{1}{4 Q}(6 C R+6 \sqrt{2 B C R Q}+B Q)$, 
$b=-\frac{P}{2 \sqrt{B R Q^{3}}}\left(2 \sqrt{2 C^{3}} R+4 C \sqrt{B R Q}+\sqrt{2 C} B Q\right), \quad c=\frac{C P^{2}}{4 B R Q^{2}}(2 C R+2 \sqrt{2 B C Q R}+B Q)$,

$A=-\frac{R}{Q^{2}}(C R-B Q)$ there is the following Bäcklund transformation between the ordinary differential Equation

(3) and the first kind of elliptic Equation (6).

$$
z^{2}(\xi)=\frac{\sqrt{B C R}\left[R+Q y^{2}(\xi)\right]}{P \sqrt{B C R}+\left[\mp\left(-C P^{2}(2 C R-2 \sqrt{2 B C R Q}+B Q)\right)^{\frac{1}{2}}+\sqrt{2 Q} C P y(\xi)\right] y(\xi)} .
$$

Case 3. When $d=-\frac{7(45+29 \sqrt{2}) B R}{4(11+6 \sqrt{2})^{2} P}, a=-\frac{(20+51 \sqrt{2}) B}{4(11+6 \sqrt{2})^{2}}, \quad b=\frac{(151+265 \sqrt{2}) B P}{28(11+6 \sqrt{2})^{2} R}$,

$c=\frac{(530+151 \sqrt{2}) B P^{2}}{196(11+6 \sqrt{2})^{2} R^{2}}, C=0, A=\frac{B R}{Q}$ there is the following Bäcklund transformation between the ordinary differential Equation (3) and the first kind of elliptic Equation (6).

$$
z^{2}(\xi)=\frac{7 R\left[R+Q y^{2}(\xi)\right]}{7 P R \mp 2(3+\sqrt{2}) \sqrt{-R P^{2} Q} y(\xi)+(1-2 \sqrt{2}) P Q y^{2}(\xi)} \quad(R Q<0) .
$$

Case 4. When $d=\frac{7(-45+29 \sqrt{2}) B R}{4(11-6 \sqrt{2})^{2} P}, a=\frac{(-20+51 \sqrt{2}) B}{4(11-6 \sqrt{2})^{2}}, \quad b=\frac{(151-265 \sqrt{2}) B P}{28(11-6 \sqrt{2})^{2} R}$,

$c=\frac{(530-151 \sqrt{2}) B P^{2}}{196(11-6 \sqrt{2})^{2} R^{2}}, C=0, A=\frac{B R}{Q}$ there is the following Bäcklund transformation between the ordinary differential Equation (3) and the first kind of elliptic Equation (6).

$$
z^{2}(\xi)=\frac{7 R\left[R+Q y^{2}(\xi)\right]}{7 P R \pm 2(-3+\sqrt{2}) \sqrt{-R P^{2} Q} y(\xi)+(1+2 \sqrt{2}) P Q y^{2}(\xi)} \quad(R Q<0) .
$$

Here $P, Q$ and $R$ are arbitrary constants not equal to zero. $A, B$ and $C$ are determined by the first kind of elliptic Equation (6).

Theorem 2.3 If $y(\xi)$ is the solution that are not the constants of the first kind of elliptic Equation (6), then the following $\bar{y}(\xi)$ is also the solution of the first kind of elliptic Equation (6).

$$
\begin{gathered}
(\bar{y}(\xi))^{2}=\frac{A\left[-B M_{0} \pm \sqrt{\left(B^{2}-4 A C\right) M_{0}^{2}}-2 C M_{0} y^{2}(\xi)\right]}{C\left[2 A M_{0} \mp\left(\mp B M_{0}+\sqrt{\left(B^{2}-4 A C\right) M_{0}^{2}}\right) y^{2}(\xi)\right]} . \\
(\bar{y}(\xi))^{2}=\frac{1}{4 C}\left[-B+2 C y^{2}(\xi) \mp 2 \sqrt{C} y^{\prime}(\xi)\right] \quad\left(B^{2}-4 A C=0\right) . \\
\bar{y}(\xi)=-\frac{A L+B L y^{2}(\xi) \mp i W \sqrt{B\left[A+B y^{2}(\xi)\right]}}{B W \pm i L \sqrt{B\left[A+B y^{2}(\xi)\right]}}(C=0, B<0) . \\
\bar{y}(\xi)=\mp \frac{H^{2}-A L^{2} y^{2}(\xi)}{L \sqrt{A^{2} L^{2}+B H^{2}} y(\xi) \mp H L \sqrt{A+B y^{2}(\xi)}} \quad(C=0) .
\end{gathered}
$$


Here $M_{0}, L, W$ and $H$ are arbitrary constants that are not all zero.

Theorem 2.4 The first kind of elliptic Equation (6) has the following some kinds of solutions.

Case 1. The Riemann $\theta$ function new solutions [7] of the first kind of elliptic equation

When $A=\theta_{4}^{2}(0) \theta_{2}^{2}(0), B=\theta_{2}^{4}(0)-\theta_{4}^{4}(0), C=-\theta_{4}^{2}(0) \theta_{2}^{2}(0)$, the first kind of elliptic Equation (6) has the following solution.

$$
y(\xi)=\frac{\theta_{1}(\xi)}{\theta_{3}(\xi)}
$$

When $A=\theta_{3}^{2}(0) \theta_{2}^{2}(0), B=-\left(\theta_{2}^{4}(0)+\theta_{3}^{4}(0)\right), C=\theta_{3}^{2}(0) \theta_{2}^{2}(0)$, the first kind of elliptic Equation (6) has the following solution.

$$
y(\xi)=\frac{\theta_{1}(\xi)}{\theta_{4}(\xi)},
$$

When $A=\theta_{4}^{2}(0) \theta_{3}^{2}(0), B=\theta_{3}^{4}(0)+\theta_{4}^{4}(0), C=\theta_{4}^{2}(0) \theta_{3}^{2}(0)$, the first kind of elliptic Equation (6) has the following solution.

$$
y(\xi)=\frac{\theta_{1}(\xi)}{\theta_{2}(\xi)}
$$

where $\theta\left(\begin{array}{c}\varepsilon \\ \varepsilon^{*}\end{array}\right)(z, \tau)=\sum_{n=-\infty}^{+\infty} \exp \left[\left(n+\frac{\varepsilon}{2}\right)\left(\pi i \tau\left(n+\frac{\varepsilon}{2}\right)+2\left(z+\frac{\varepsilon^{*}}{2}\right)\right)\right], \quad\left(\begin{array}{c}\varepsilon \\ \varepsilon^{*}\end{array}\right)$ is a bivector, $n$ is a constant. And, $\theta_{1}(z)=\theta\left(\begin{array}{l}1 \\ 1\end{array}\right)(z ; \tau), \theta_{2}(z)=\theta\left(\begin{array}{l}1 \\ 0\end{array}\right)(z ; \tau), \theta_{3}(z)=\theta\left(\begin{array}{l}0 \\ 0\end{array}\right)(z ; \tau), \theta_{4}(z)=\theta\left(\begin{array}{l}0 \\ 1\end{array}\right)(z ; \tau)$.

Case 2. The Jacobi elliptic function new solutions of the first kind of elliptic equation

According to the periodicity of the Jacobi elliptic function, some kinds of new solutions of the first kind of elliptic equation are obtained, some new solutions [6] [8] [9] are listed here.

When $A=1, B=-1-k^{2}, C=k^{2}$, the first kind of elliptic Equation (6) has the following solutions.

$$
\begin{aligned}
& y(\xi)=\operatorname{sn}(\xi, k), \\
& y(\xi)=\left\{\begin{array}{lc}
\operatorname{sn}(\xi, k), & (4 p-1) K(k) \leq \xi \leq(4 p+3) K(k) \quad p \in Z, \\
-1, & \text { others. }
\end{array}\right. \\
& y(\xi)= \begin{cases}1, & \xi \leq(4 p+1) K(k), \\
\operatorname{sn}(\xi, k), & (4 p+1) K(k) \leq \xi \leq(4 p+3) K(k), \\
-1, & (4 p+3) K(k) \leq \xi \quad p \in Z .\end{cases} \\
& y(\xi)= \begin{cases}-1, & \xi \leq(4 p+3) K(k), \\
\operatorname{sn}(\xi, k), & (4 p+3) K(k) \leq \xi \leq(4 p+5) K(k), \\
1, & (4 p+5) K(k) \leq \xi \quad p \in Z .\end{cases}
\end{aligned}
$$

When $A=1-k^{2}, B=2 k^{2}-1, C=-k^{2}$, the first kind of elliptic Equation (6) has the following solutions.

$$
\begin{gathered}
y(\xi)=\operatorname{cn}(\xi, k), \\
y(\xi)= \begin{cases}\operatorname{cn}(\xi, k), & (4 p+2) K(k) \leq \xi \leq(4 p+6) K(k) \quad p \in Z, \\
-1, & \text { others. }\end{cases} \\
y(\xi)= \begin{cases}1, & \xi \leq 4 p K(k), \\
\operatorname{cn}(\xi, k), & 4 p K(k) \leq \xi \leq(4 p+2) K(k), \\
-1, & \xi \geq(4 p+2) K(k) \quad p \in Z .\end{cases}
\end{gathered}
$$




$$
y(\xi)= \begin{cases}-1, & \xi \leq(4 p+2) K(k) \\ \operatorname{cn}(\xi, k), & (4 p+2) K(k) \leq \xi \leq(4 p+4) K(k) \\ 1, & \xi \geq(4 p+4) K(k) \quad p \in Z\end{cases}
$$

When $A=-1+k^{2}, B=2-k^{2}, C=-1$, the first kind of elliptic Equation (6) has the following solutions.

$$
\begin{aligned}
& y(\xi)=\operatorname{dn}(\xi, k), \\
& y(\xi)=\left\{\begin{array}{lc}
\operatorname{dn}(\xi, k), & 2 p K(k) \leq \xi \leq(2 p+2) K(k) \quad p \in Z, \\
1, & \text { others. }
\end{array}\right. \\
& y(\xi)=\left\{\begin{array}{lc}
\operatorname{dn}(\xi, k), & (2 p+1) K(k) \leq \xi \leq(2 p+3) K(k) \quad p \in Z, \\
\sqrt{1-k^{2}}, & \text { others. }
\end{array}\right. \\
& y(\xi)= \begin{cases}\sqrt{1-k^{2}}, & \xi \leq(2 p+1) K(k), \\
\operatorname{dn}(\xi, k), & (2 p+1) K(k) \leq \xi \leq(2 p+2) K(k), \\
1, & \xi \geq(2 p+2) K(k) \quad p \in Z .\end{cases} \\
& y(\xi)= \begin{cases}1, & \xi \leq(2 p+2) K(k), \\
\operatorname{dn}(\xi, k), & (2 p+2) K(k) \leq \xi \leq(2 p+3) K(k), \\
\sqrt{1-k^{2}}, & \xi \geq(2 p+3) K(k) \quad p \in Z .\end{cases}
\end{aligned}
$$

where $K(k)=\int_{0}^{\frac{\pi}{2}} \frac{1}{\sqrt{1-k^{2} \sin ^{2} \varphi}} \mathrm{d} \varphi=\int_{0}^{1} \frac{1}{\sqrt{\left(1-x^{2}\right)\left(1-k^{2} x^{2}\right)}} \mathrm{d} x, 0 \leq k \leq 1 . \quad Z$ is the integer assemblage.

Case 3. The other new solutions of the first kind of elliptic equation

When $B^{2}-4 A C=0$, the first kind of elliptic Equation (6) has the following solutions.

$$
\begin{gathered}
y(\xi)=\frac{\sqrt{B}}{\sqrt{2 C}} \tan \left(\frac{\sqrt{B}}{\sqrt{2}}|\xi|\right) \quad(B>0, C>0), \\
y(\xi)=\frac{\sqrt{-B}[1+\exp (\sqrt{-2 B}|\xi|)]}{\sqrt{2 C}[1-\exp (\sqrt{-2 B}|\xi|)]} \quad(B<0, C>0) .
\end{gathered}
$$

\section{The New Infinite Sequence Solutions of Two Kinds of Sine-Gordon Equations}

\subsection{The New Infinite Sequence Solutions of the Treble Sine-Gordon Equations}

Substituting the functional transformation $u(x, t)=u(\xi)=6 \arctan (v(\xi)), \xi=\mu x+\omega t$ (where $\mu$ and $\omega$ are undetermined constants) into the Equation (2) yields the following ordinary differential equation

$$
\begin{aligned}
v^{\prime \prime}(\xi)= & -\frac{1}{9\left(\mu^{2}-\omega^{2}\right)\left[1+v^{2}(\xi)\right]^{2}}\left[(\alpha+4 \beta+9 \gamma) v(\xi)+2(\alpha-15 \gamma) v^{3}(\xi)\right. \\
& \left.+(\alpha-4 \beta+9 \gamma) v^{5}(\xi)-18\left(\mu^{2}-\omega^{2}\right)\left[v(\xi)+v^{3}(\xi)\right]\left(v^{\prime}(\xi)\right)^{2}\right] .
\end{aligned}
$$

By the functional transformation, the ordinary differential Equation (33) is changed to the ordinary differential Equations (34) 


$$
\left\{\begin{aligned}
\frac{\mathrm{d} v}{\mathrm{~d} \xi}= & Y, \\
\frac{\mathrm{d} Y}{\mathrm{~d} \xi}= & -\frac{1}{9\left(\mu^{2}-\omega^{2}\right)\left[1+v^{2}(\xi)\right]^{2}}\left[(\alpha+4 \beta+9 \gamma) v(\xi)+2(\alpha-15 \gamma) v^{3}(\xi)\right. \\
& \left.+(\alpha-4 \beta+9 \gamma) v^{5}(\xi)-18\left(\mu^{2}-\omega^{2}\right)\left[v(\xi)+v^{3}(\xi)\right] Y^{2}\right] .
\end{aligned}\right.
$$

Then by the functional transformation (35), the ordinary differential Equations (34) is changed to the ordinary differential Equations (36)

$$
\begin{gathered}
\mathrm{d} \xi=9\left(\mu^{2}-\omega^{2}\right)\left(1+v^{2}\right) \mathrm{d} \tau \\
\left\{\begin{array}{l}
\frac{\mathrm{d} v}{\mathrm{~d} \tau}=9\left(\mu^{2}-\omega^{2}\right)\left(1+v^{2}\right) Y \\
\frac{\mathrm{d} Y}{\mathrm{~d} \tau}=-\frac{1}{1+v^{2}}\left[(\alpha+4 \beta+9 \gamma) v+2(\alpha-15 \gamma) v^{3}+(\alpha-4 \beta+9 \gamma) v^{5}\right. \\
\left.-18\left(\mu^{2}-\omega^{2}\right)\left(v+v^{3}\right) Y^{2}\right]
\end{array}\right.
\end{gathered}
$$

We can obtain the first integral of the ordinary differential Equations (36) as follows

$$
Y^{2}=\frac{1}{9\left(\mu^{2}-\omega^{2}\right)\left(1+v^{2}\right)}\left[\gamma\left(1-3 v^{2}\right)^{2}+\alpha\left(1+v^{2}\right)^{2}+\left(1+v^{2}\right)\left[-4 \beta v^{2}+9 C_{0}\left(\mu^{2}-\omega^{2}\right)\left(1+v^{2}\right)^{2}\right]\right],
$$

where $C_{0}$ is an arbitrary constant.

Substituting the first integral (37) into the first equation of the ordinary differential Equations (36) yields the following ordinary differential equation

$$
\left(\frac{\mathrm{d} v}{\mathrm{~d} \tau}\right)^{2}=L_{0}+L_{1} v^{2}+L_{2} v^{4}+L_{3} v^{6}+L_{4} v^{8}
$$

where $L_{0}=9\left(\mu^{2}-\omega^{2}\right)\left[\alpha+\gamma+9 C_{0}\left(\mu^{2}-\omega^{2}\right)\right], \quad L_{1}=9\left(\mu^{2}-\omega^{2}\right)\left[3 \alpha-4 \beta-5 \gamma+36 C_{0}\left(\mu^{2}-\omega^{2}\right)\right]$, $L_{2}=9\left(\mu^{2}-\omega^{2}\right)\left[3 \alpha-8 \beta+3 \gamma+54 C_{0}\left(\mu^{2}-\omega^{2}\right)\right], \quad L_{3}=9\left(\mu^{2}-\omega^{2}\right)\left[\alpha-4 \beta+9\left(\gamma+4 C_{0}\left(\mu^{2}-\omega^{2}\right)\right)\right]$, $L_{4}=81 C_{0}\left(\mu^{2}-\omega^{2}\right)^{2}$.

With the help of the relative conclusions of some kinds of ordinary differential equations introduced in Part 2, the new infinite sequence solutions of the treble sine-Gordon equations are constructed

\subsubsection{To Construct the New Infinite Sequence Solutions When $C_{0}=0$}

When $C_{0}=0$, the ordinary differential Equations (38) is changed to the following the ordinary differential equation.

$$
\left(\frac{\mathrm{d} v}{\mathrm{~d} \tau}\right)^{2}=L_{10}+L_{11} v^{2}+L_{12} v^{4}+L_{13} v^{6} .
$$

where $L_{10}=9\left(\mu^{2}-\omega^{2}\right)(\alpha+\gamma), \quad L_{11}=9\left(\mu^{2}-\omega^{2}\right)(3 \alpha-4 \beta-5 \gamma), \quad L_{12}=9\left(\mu^{2}-\omega^{2}\right)(3 \alpha-8 \beta+3 \gamma)$, $L_{13}=9\left(\mu^{2}-\omega^{2}\right)(\alpha-4 \beta+9 \gamma)$.

Case 1. The new infinite sequence smooth-type soliton-like solutions

When the coefficients of the ordinary differential Equation (39) $L_{10}=d, L_{11}=a, L_{12}=b$ and $L_{13}=c$ satisfy the case 2. of theorem 2.2, with the help of the following superposition formula, the new infinite sequence smooth-type soliton-like solutions of the treble sine-Gordon equations are obtained, which are consisting of Riemann $\theta$ function, Jacobi elliptic function and hyperbolic function. 


$$
\left\{\begin{array}{l}
u_{n}(x, t)=u_{n}(\xi)=6 \arctan \left(v_{n}(\xi)\right) \quad(n=0,1,2, \cdots), \\
\mathrm{d} \xi=9\left(\mu^{2}-\omega^{2}\right)\left(1+v_{n}^{2}\right) \mathrm{d} \tau, \\
v_{n}^{2}(\tau)=\frac{\sqrt{B C R}\left[R+Q y_{n}^{2}(\tau)\right]}{P \sqrt{B C R}+\left[\mp\left(-C P^{2}(2 C R-2 \sqrt{2 B C R Q}+B Q)\right)^{\frac{1}{2}}+\sqrt{2 Q} C P y_{n}(\tau)\right] y_{n}(\tau)} \\
\left(y_{n}(\tau)\right)^{2}=\frac{A\left[-B M_{0} \pm \sqrt{\left(B^{2}-4 A C\right) M_{0}^{2}}-2 C M_{0} y_{n-1}^{2}(\tau)\right]}{C\left[2 A M_{0} \mp\left(\mp B M_{0}+\sqrt{\left(B^{2}-4 A C\right) M_{0}^{2}}\right) y_{n-1}^{2}(\tau)\right]} \quad(n=1,2, \cdots) .
\end{array}\right.
$$

where $y_{0}(\tau)$ is determined by the first kind of elliptic Equation (6). $A, B$ and $C$ are the coefficients of the first kind of elliptic Equation (6), and $A=-\frac{R}{Q^{2}}(C R-B Q) . P, Q$ and $R$ are arbitrary constants that are not all zero.

If the $y_{0}(\tau)$ of the superposition Formula (40) are taken as the Solutions (15)-(17) of the first kind of elliptic Equation (6), then the Riemann $\theta$ function type new infinite sequence solutions of the treble sine-Gordon equations are obtained.

If the $y_{0}(\tau)$ of the superposition Formula (40) are taken as the Solutions (18)-(30) of the first kind of elliptic Equation (6), then the Jacobi elliptic function type new infinite sequence smooth-type soliton-like and tight-soliton solutions of the treble sine-Gordon equations are obtained.

If $k=1$ in the Jacobi elliptic function type solutions of the treble sine-Gordon equations, then the hyperbolic function type new infinite sequence solutions are obtained.

Case 2. The new infinite sequence peak-type soliton solutions

When $L_{10}=d, L_{11}=a, L_{12}=b$ and $L_{13}=c$ satisfy the Case 2. of the Theorem 2.2, with the help of the following superposition formula, the new infinite sequence peak soliton-like solutions of the treble sine-Gordon equations are obtained, which are consisting of hyperbolic function and trigonometric function.

$$
\left\{\begin{array}{l}
u_{n}(x, t)=u_{n}(\xi)=6 \arctan \left(v_{n}(\xi)\right) \quad(n=0,1,2, \cdots), \\
\mathrm{d} \xi=9\left(\mu^{2}-\omega^{2}\right)\left(1+v_{n}^{2}\right) \mathrm{d} \tau, \\
v_{n}^{2}(\tau)=\frac{\sqrt{B C R}\left[R+Q y_{n}^{2}(\tau)\right]}{P \sqrt{B C R}+\left[\mp\left(-C P^{2}(2 C R-2 \sqrt{2 B C R Q}+B Q)\right)^{\frac{1}{2}}+\sqrt{2 Q} C P y_{n}(\tau)\right] y_{n}(\tau)} \\
\left(y_{n}(\tau)\right)^{2}=\frac{1}{4 C}\left[-B+2 C y_{n-1}^{2}(\tau) \mp 2 \sqrt{C} y_{n-1}^{\prime}(\tau)\right] \quad\left(B^{2}-4 A C=0, n=1,2, \cdots\right) .
\end{array}\right.
$$

where $y_{0}(\tau)$ is determined by the first kind of elliptic Equation (6). $A, B$ and $C$ are the coefficients of the first kind of elliptic Equation (15), and $A=-\frac{R}{Q^{2}}(C R-B Q) . P, Q$ and $R$ are arbitrary constants that are not all zero.

If the $y_{0}(\tau)$ of the superposition Formula (41) is taken as the Solution (31), then the trigonometric function type new infinite sequence solutions of the treble sine-Gordon equations are obtained.

If the $y_{0}(\tau)$ of the superposition Formula (41) is taken as the Solution (32), then the hyperbolic function type new infinite sequence solutions of the treble sine-Gordon equations are obtained.

\subsubsection{To Construct the New Infinite Sequence Solutions When $C_{0}=0, \gamma=-\alpha$}

The ordinary differential Equations (38) is changed to the following the ordinary differential equation when $C_{0}=0, \gamma=-\alpha$.

$$
\left(\frac{\mathrm{d} v}{\mathrm{~d} \tau}\right)^{2}=L_{21} v^{2}+L_{22} v^{4}+L_{23} v^{6}
$$


where $L_{21}=9\left(\mu^{2}-\omega^{2}\right)(8 \alpha-4 \beta), L_{22}=-72\left(\mu^{2}-\omega^{2}\right) \beta, L_{23}=9\left(\mu^{2}-\omega^{2}\right)(-4 \beta-8 \alpha)$.

Equation (42) is changed to the Riccati Equation (44) with the help of the following functional transformation

$$
\begin{gathered}
v^{2}(\tau)=-\frac{-36(2 \alpha-\beta)\left(\mu^{2}-\omega^{2}\right)+H^{2}(\tau)}{12\left[6 \beta\left(\mu^{2}-\omega^{2}\right)+\sqrt{-(2 \alpha+\beta)\left(\mu^{2}-\omega^{2}\right)} H(\tau)\right]} . \\
\frac{\mathrm{d} H(\tau)}{\mathrm{d} \tau}=-36(2 \alpha-\beta)\left(\mu^{2}-\omega^{2}\right)+H^{2}(\tau) .
\end{gathered}
$$

By the following superposition formula, the new infinite sequence soliton-like solutions of the treble sine-Gordon equations are obtained, which are consisting of hyperbolic function, trigonometric function and rational function.

$$
\left\{\begin{array}{l}
u_{n}(x, t)=u_{n}(\xi)=6 \arctan \left(v_{n}(\xi)\right) \quad(n=0,1,2, \cdots), \\
\mathrm{d} \xi=9\left(\mu^{2}-\omega^{2}\right)\left(1+v_{n}^{2}\right) \mathrm{d} \tau \\
v_{n}^{2}(\tau)=-\frac{-36(2 \alpha-\beta)\left(\mu^{2}-\omega^{2}\right)+H_{n}^{2}(\tau)}{12\left[6 \beta\left(\mu^{2}-\omega^{2}\right)+\sqrt{-(2 \alpha+\beta)\left(\mu^{2}-\omega^{2}\right)} H_{n}(\tau)\right]}, \\
H_{n}(\tau)=\frac{-a\left[-r H_{n-3}(\tau)+(p+r) H_{n-2}(\tau)-p H_{n-1}(\tau)\right]}{-r H_{n-2}(\tau) H_{n-1}(\tau)+H_{n-3}(\tau)\left[-p H_{n-2}(\tau)+(p+r) H_{n-1}(\tau)\right]} \quad(n=4,5, \cdots) .
\end{array}\right.
$$

where $p$ and $r$ are arbitrary constants that are not all zero. $a=36(2 \alpha-\beta)\left(\mu^{2}-\omega^{2}\right)$. And $H_{1}(\tau), H_{2}(\tau)$ and $H_{3}(\tau)$ are the solutions of the Riccati Equation (44).

3.1.3. To Construct the New Infinite Sequence Solutions When $C_{0}=0, \alpha=4 \beta-9 \gamma$

When $C_{0}=0, \alpha=4 \beta-9 \gamma$, the ordinary differential Equations (38) is changed to the following the ordinary differential equation.

$$
\left(\frac{\mathrm{d} v}{\mathrm{~d} \tau}\right)^{2}=L_{31}+L_{32} v^{2}+L_{33} v^{4}
$$

where $L_{31}=36\left(\mu^{2}-\omega^{2}\right)(\beta-2 \gamma), L_{32}=36\left(\mu^{2}-\omega^{2}\right)(2 \beta-8 \gamma), L_{33}=36\left(\mu^{2}-\omega^{2}\right)(\beta-6 \gamma)$.

$$
\left\{\begin{array}{l}
u_{n}(x, t)=u_{n}(\xi)=6 \arctan \left(v_{n}(\xi)\right) \quad(n=0,1,2, \cdots), \\
\mathrm{d} \xi=9\left(\mu^{2}-\omega^{2}\right)\left(1+v_{n}^{2}\right) \mathrm{d} \tau, \\
v_{n}^{2}(\tau)=\frac{A\left[-B M_{0} \pm \sqrt{\left(B^{2}-4 A C\right) M_{0}^{2}}-2 C M_{0} v_{n-1}^{2}(\tau)\right]}{C\left[2 A M_{0} \mp\left(\mp B M_{0}+\sqrt{\left(B^{2}-4 A C\right) M_{0}^{2}}\right) v_{n-1}^{2}(\tau)\right]} \quad(n=1,2, \cdots) .
\end{array}\right.
$$

where $A=L_{31}, B=L_{32}, C=L_{33} ; M_{0}$ is an arbitrary constants that is not zero. $v_{0}(\tau)$ is determined by the first kind of elliptic Equation (46).

\subsection{The New Infinite Sequence Solutions of the Double Sine-Gordon Equations}

Substituting the functional transformation $u(x, t)=u(\xi)=2 \arctan (v(\xi)), \xi=\mu x+\omega t$ (where $\mu$ and $\omega$ are undetermined constants) into Equation (1) yields the following ordinary differential equation

$$
v^{\prime \prime}(\xi)=\frac{1}{\mu \omega\left[1+v^{2}(\xi)\right]}\left[(p+4 p \lambda) v(\xi)+(p-4 p \lambda) v^{3}(\xi)+2 \mu \omega v(\xi)\left(v^{\prime}(\xi)\right)^{2}\right] .
$$

By the functional transformation, the ordinary differential Equation (48) is changed to the following two 
ordinary differential equations

$$
\begin{aligned}
& \left\{\begin{array}{l}
\frac{\mathrm{d} v}{\mathrm{~d} \xi}=Y, \\
\frac{\mathrm{d} Y}{\mathrm{~d} \xi}=\frac{1}{\mu \omega\left(1+v^{2}\right)}\left[(p+4 p \lambda) v+(p-4 p \lambda) v^{3}+2 \mu \omega v Y^{2}\right] .
\end{array}\right. \\
& \left\{\begin{array}{l}
\frac{\mathrm{d} v}{\mathrm{~d} \tau}=\mu \omega\left(1+v^{2}\right) Y, \\
\frac{\mathrm{d} Y}{\mathrm{~d} \tau}=(p+4 p \lambda) v+(p-4 p \lambda) v^{3}+2 \mu \omega v Y^{2} \\
\mathrm{~d} \xi=\mu \omega\left(1+v^{2}\right) \mathrm{d} \tau .
\end{array}\right.
\end{aligned}
$$

The two ordinary differential equations have the following first integral

$$
Y^{2}=\frac{1}{\mu \omega}\left[p\left[-1+(-1+4 \lambda) v^{2}\right]+C_{0} \mu \omega\left(1+v^{2}\right)^{2}\right] .
$$

where $C_{0}$ is an integral constant.

Substituting the first integral into the first equation of the ordinary differential Equations (49) and (50) severally yields the following two ordinary differential equation

$$
\begin{gathered}
\left(\frac{\mathrm{d} v}{\mathrm{~d} \xi}\right)^{2}=C_{0} v^{4}+\left(2 C_{0}-\frac{p}{\mu \omega}+\frac{4 p \lambda}{\mu \omega}\right) v^{2}+C_{0}-\frac{p}{\mu \omega} . \\
\left(\frac{\mathrm{d} v}{\mathrm{~d} \tau}\right)^{2}=K_{0} v^{8}+K_{1} v^{6}+K_{2} v^{4}+K_{3} v^{2}+K_{4} .
\end{gathered}
$$

where $K_{0}=C_{0} \mu^{2} \omega^{2}, K_{1}=(-p+4 p \lambda) \mu \omega+4 C_{0} \mu^{2} \omega^{2}, K_{2}=(-3+8 \lambda) p \mu \omega+6 C_{0} \mu^{2} \omega^{2}$, $K_{3}=(-3+4 \lambda) p \mu \omega+4 C_{0} \mu^{2} \omega^{2}, K_{4}=-p \mu \omega+C_{0} \mu^{2} \omega^{2}$

When $C_{0}=0$, the ordinary differential Equations (53) is changed to the following the ordinary differential equation.

$$
\left(\frac{\mathrm{d} v}{\mathrm{~d} \tau}\right)^{2}=K_{11} v^{6}+K_{12} v^{4}+K_{13} v^{2}+K_{14} .
$$

where $K_{11}=(-p+4 p \lambda) \mu \omega, K_{12}=(-3+8 \lambda) p \mu \omega, K_{13}=(-3+4 \lambda) p \mu \omega, K_{14}=-p \mu \omega$.

By the method to construct the new infinite sequence solutions of the treble sine-Gordon equation, we can also obtain the new infinite sequence solutions of the double sine-Gordon equation (not given here).

\section{Conclusion}

By the auxiliary equation method, many kinds of smooth type soliton, tense type soliton and peak soliton and so on new solutions of the nonlinear evolution equations have been obtained [1]-[14]. In this paper, by the function transformation and the first integral of the ordinary differential equations, the new infinite sequence soliton-like solutions consisting of the Riemann $\theta$ function, Jacobi elliptic function, hyperbolic function, trigonometric function and rational function of the double sine-Gordon equation and the treble sine-Gordon equation are obtained. These solutions conclude many kinds of smooth type soliton, tense type soliton and peak soliton and so on new solutions. Refs. [1]-[5] didn't obtain the new tense type soliton and peak soliton solutions of the double sine-Gordon equation and the treble sine-Gordon equation.

\section{Acknowledgements}

Project supported by the Natural Science Foundation of China (Grant No. 11361040), the Science Research 
Foundation of Institution of Higher Education of Inner Mongolia Autonomous Region, China (Grant No. NJZY16180) and the Natural Science Foundation of Inner Mongolia Autonomous Region, China (Grant No. 2015MS0128).

\section{References}

[1] Sirendaoerji and Jiong, S. (2002) A Direct Method for Solving Sine-Gordon Type Equations. Physics Letters A, 298, 133-139.

[2] Xie, Y.X. and Tang, J.S. (2005) A Unified Approach in Seeking the Solitary Wave Solutions to Sine-Gordon Type Equations. Chinese Physics, 14, 1303-1306. http://dx.doi.org/10.1088/1009-1963/14/7/006

[3] Zheng, Q. and Ren, Z.Z. (2008) Some New Exact Traveling Wave Solutions of Double-Sine-Gordon Equation. Communications in Theoretical Physics, 49, 303-304. http://dx.doi.org/10.1088/0253-6102/49/2/09

[4] Yang, J.S. and Lou, S.Y. (2004) Solitary Wave Solutions of Triple Sine-Gordon Equation. Chinese Physics Letters, 21, 608-611. http://dx.doi.org/10.1088/0256-307X/21/4/005

[5] Liu, C.S. (2004) Travelling Wave Solutions of Triple Sine-Gordon Equation. Chinese Physics Letters, 21, $2369-2371$. http://dx.doi.org/10.1088/0256-307X/21/12/014

[6] Taogetusang and Yi, L.N. (2014) New Infinite Sequence Solutions to Equations of Sine-Gordon Type. Acta Physica Sinica, 63, Article ID: 215202.

[7] Wang, J.M. (2012) Riemann $\theta$ Function Solutions to Modified Korteweg de Vries-Sine-Gordon Equation. Acta Physica Sinica, 61, Article ID: 080201.

[8] Taogetusang and Yi, L.N. (2014) New Complexion Two-Soliton Solutions to a Kind of Nonlinear Coupled System. Acta Physica Sinica, 63, Article ID: 160201.

[9] Taogetusang, Sirendaoerji and Li, S.M. (2010) New Application to Riccati Equation. Chinese Physics B, 19, Article ID: 080303. http://dx.doi.org/10.1088/1674-1056/19/8/080303

[10] Rui, W.G. (2013) Different Kinds of Exact Solutions with Two-Loop Character of the Two-Component Short Pulse Equations of the First Kind. Communications in Nonlinear Science and Numerical Simulation, 18, 2667-2678. http://dx.doi.org/10.1016/j.cnsns.2013.01.020

[11] Khaled, A. and Gepreel, S.O. (2012) Exact Solutions for Nonlinear Partial Fractional Differential Equations. Chinese Physics B, 21, Article ID: 110204. http://dx.doi.org/10.1088/1674-1056/21/11/110204

[12] Chen, Y. and Fan, E.G. (2007) Complexiton Solutions of the (2+1)-Dimensional Dispersive Long Wave Equation. Chinese Physics, 16, 6-15. http://dx.doi.org/10.1088/1009-1963/16/1/002

[13] Zhu, W.T., Ma, S.H., Fang, J.P., Ma, Z.Y. and Zhu, H.P. (2014) Fusion, Fission, and Annihilation of Complex Waves for the (2+1)-Dimensional Generalized Calogero-Bogoyavlenskii-Schiff System. Chinese Physics B, 23, Article ID: 060505. http://dx.doi.org/10.1088/1674-1056/23/6/060505

[14] Chen, W.L., Zhang, W.T., Zhang, L.P. and Dai, C.Q. (2013) Interaction Behaviors between Special Dromions the (2+1)-Dimensional Broer-Kaup-Kupershmidt Equation. Communications in Theoretical Physics, 59, 68-72. http://dx.doi.org/10.1088/0253-6102/59/1/13 